\title{
Operads and Quantum Gravity
}

\author{
Ioannis P. ZOIS* \\ IHES, Le Bois-Marie, 35, route de Chartres \\ F-91440 Bures-sur-Yvette, FRANCE \\ and \\ Mathematical Institute, Oxford University \\ 24-29 St. Giles', Oxford, OX1 3LB, UK
}

"In girum imus nocte et consumimur igni".

\begin{abstract}
In this article we try to explain and extend a statement due to Maxim Kontsevich back in 1999, that the Holography Principle in physics should be related to the (higher dimensional) Deligne Conjecture in mathematics. This seems to suggest that the little d-discs operad (or equivalently the notion of a $d$-algebra) gives a new way to understand the mathematical aspects of quantum gravity using holography. The strategy is as follows: we would like to learn something about quantum gravity in $(d+1)$ dimensions: we use holography to reduce our original problem to a CFT in $d$-dimensions. The deep origin of this dimensional reduction lies on the fact that it is the area and not the volume which appears in the formula giving the entropy of black holes as described long ago by Hawking. Then we use $d$-algebras (i.e. the little $d$-discs operad) to study our $d$-dim CFT. The possible relation between $d$-dim CFT and $d$-algebras comes from the lesson we have learnt from strings (namely the 2-dim CFT case): the space of physical states in closed string field theory (ie the BRST cohomology) has a natural Gerstenhaber algebra structure and this by Cohen's theorem is related to the little 2-discs operad. The proposal then is that the relation might hold in higher than 2 dimensions. This approach is algebraic although it would have been much more satisfactory if we
\end{abstract}

*izois@ihes.fr Current address: School of Mathematics, Cardiff University, PO Box 926, Cardiff CF24 4YH, UK; e-mail address: zoisip@cf.ac.uk 
could generalise Segal's geometric approach to CFT in higher than 2 dimensions. Hopefully the article is mathematically self-contained.

PACS classification: 11.10.-z; 11.15.-q; 11.30.-Ly

Keywords: Holography, String Theory, M-Theory, Quantum Gravity, Conformal Field Theory, Operads, Motives.

\section{Introduction}

This work was motivated by an attempt to understand an interesting statement by Maxim Kontsevich [7] back in 1999: that the Holography Principle originally due to G. 't Hooft [2] in Physics might be related to the Higher Dimensional Deligne Conjecture in Mathematics due to Kontsevich. In 2000 Kontsevich and Soibelman [8] proved the original Deligne conjecture. A secong proof was given by Tamarkin (see [9]). Very recently yet another proof of the Deligne conjecture appeared which is due to A. Kohlhuber using the arc operad.

Let us roughly explain both these statements here and elaborate more on them later: Holography is a statement about quantum gravity; in simple terms it says that quantum gravity must be a topological quantum field theory.

On the other hand the original Deligne conjecture (due to Deligne as the name suggests, see [3]), it is about the Hochschild complex of associative algebras; as it is well-known the Hochschild complex is very useful when one wants to study the theory of deformations of algebras.

We organise this article as follows: in section 2 we try to explain the necessity for holography in physics starting from black hole puzzles; in section 3 we give the necessary mathematical definitions about operads and Gerstenhaber algebras and we also state the two basic theorems: the first is due to F. Cohen and relates Gerstenhaber algebras with the little 2-discs operad and the second is D. Tamarkin's theorem on the formality of the little $d$-discs operad. In the last section we explain the relation between string theory and the little 2-discs operad. Then as we shall see, one will be able to interpret Kontsevich's statement as a higher dimensional analogue of the above relation. 
We start our discussion with possibly the most mysterious objects in the universe: Black Holes.

\section{Understanding Black Holes from Strings: the need for a nonlocality mechanism}

We have gained some understanding on two important problems in black hole physics (abreviated to "BH" in the sequel) by using some recent results from string theory dualities (for more details one can see [1] which is a nice review article):

1. In general relativity we have the so-called "no hair" theorem which refers to black holes. This is the statement that the configuration of a black hole solution given by the Schwarzchild metric is uniquely determined by its mass (= total energy) (we assume no more conserved quantities like electric charge or angular momentum for simplicity). In other words we have only one configuration (for a given mass) associated to the Schwarzchild solution.

Following the usual definition for the classical entropy of a system

$$
S=k_{B} \ln \Omega
$$

where $\Omega$ is the number of microstates compatible with some given values of the macroscopic parameters (eg temperature, pressure, volume etc) and $k_{B}$ is Boltzmann's constant, we immediately deduce that a BH must have zero entropy classically since $\ln 1=0$.

But then we encounter the qualitative argument originally due to Beckenstein that if this was indeed the case, then any object, e.g. some gas, falling into a $\mathrm{BH}$ would contradict the second law of thermodynamics. To avoid that one should associate a nonzero entropy (positive of course) to any $\mathrm{BH}$. The precise value of the entropy $S$ of a $\mathrm{BH}$ was then determined by the Hawking area formula which, ignoring constants, reads (see for example [16])

$$
S \sim A
$$

where $A$ is the area of the event horizon. So if we denote by $R$ the radius of the event horizon of a $\mathrm{BH}$, its entropy is proportional to $R^{2}$ and not proportional to $R^{3}$. The origin of $\mathrm{BH}$ entropy was understood to be quantum 
mechanical.

At this point we should make a comment: the Hawking formula is quite surprising, remarkable and counterintuitive since from what we know from thermodynamics the entropy of a system depends on its volume but in this case of black holes a dimensional reduction occures and in fact the entropy is proportional only to the area of its event horizon.

Nonetheless we know that statistical physics gives a more fundamental explanation of the laws of thermodynamics and moreover a correct "wouldbe" quantum theory of gravity should explain the origin of the quantum states associated to a $\mathrm{BH}$. So the challenge was to find a statistical explanation for the quantum states associated to a $\mathrm{BH}$ which give rise to its entropy described by the Hawking formula.

Superstring theory can indeed, in some cases, provide an explanation for the origin of quantum states associated to multicharged extremal black holes. And anyway string theory is arguably the best known candidate for a quantum theory of gravity. The argument which explains the microscopic origin of BH entropy starting from string theory was originally due to Strominger, Vafa, Horowitz and Maldacena and it is based on S-duality. The later is a statement about an isomorphism between strong and weak coupling regions of superstring theory; equivalently it interchanges monopoles with charges in the theory (or equivalently it interchanges topology and dynamics) and gives us the ability to identify BPS superstring states which will either be perturbative states if they carry $N S$ charges or $D$-branes if they carry $R$ charges in "weak coupling" region with extremal black holes carrying the analogous type of charge in "strong coupling" region. We restrict our attention to BPS states (these are states whose mass does not receive any quantum corrections) because for simplicity we assume no backcreation for the black hole (namely its mass which is equal to its energy remains constant). Briefly then the main idea behind this string theoretic explanation of the quantum states associated to a black hole is that since superstrings live in 10-dim and $\mathrm{BH}$ in 4-dim, the remaining 6 compactified dimensions essentially provide a "phase space" which we quantize and thus we obtain the states of the BH. (This picture is not utterly correct but we think captures the spirit of the argument and gives a clear picture conceptually). For a possible noncommutative generalisation of the Beckenstein-Hawking areaentropy formula for BHs see [12]. 
2. The second problem we would like to consider in BH physics is the so-called "information paradox". Classically, nothing can escape the event horizon of a $\mathrm{BH}$ (since that would require a velocity which is grater than the velocity of light; one can use that as a definition of the "event horizon"). Yet quantum mechanically, since a $\mathrm{BH}$ has a positive entropy as we just argued above, assuming a thermodynamical behaviour, it should also have a corresponding temperature from the well-known relation in thermodynamics

$$
(d M=) d E=T d S .
$$

This is the Hawking temperature

$$
T_{H}=\frac{\hbar \kappa}{2 k_{B} \pi}
$$

where $\kappa$ is surface gravity (the acceleration felt by a static object at the horizon as measured from the asymptotic region), and hence BH's should also radiate.

This is the Hawking radiation. Then the problem with radiation carrying out the information of formation, assuming no quantum xeroxing, is to maintain unitarity of the process as ordinary quantum mechanics requires. More precisely, the way one computes Hawking radiation is by assuming a codim-1 foliation of spacetime where the normal direction is time and this radiation process appears to be nonunitary. (For a more general discussion about foliations and the relation between physical and topological entropy one can see [11] and [12]).

Thus trying to avoid a classical contradiction with the 2nd law of thermodynamics we assumed that BH's have positive entropy (whose origin is quantum mechanical); yet this almost immediately created another contradiction with quantum mechanics: loss of unitarity in $\mathrm{BH}$ radiation. Unitarity is absolutely crucial in any quantum theory since it reflects the conservation of probabilities. So it seems that we didn't actually achieve very much: we simply "pushed" the contradiction from the classical to the quantum realm.

It appears that the most economical (i.e. requiring the fewest changes to things we already know in physics) way out is to assume that there is some physical principle which does not allow this to happen. We enforce unitarity 
throughout by imposing a non-locality mechanism. One such mechanism is the holography principle due to G. 't Hooft (see [2]). The original statement is the following:

"A quantum theory of gravity on a $(d+1)$-manifold with boundary should be equivalent to a conformal field theory (CFT for short) on the boundary (which is a d-manifold) and this conformal field theory on the boundary must have one degree of freedom per Planck area".

Let us elaborate more on this: since for BH we seem to lose any information passing the event horizon, it is reasonable to assume that in order to avoid this problem (along with its quantum mechanical incarnation of nonunitary radiation), everything that happens inside the black hole should be described from data on its event horizon. It is clear we think that the motivation for this dimensional reduction of quantum gravity in holography came from the formula for the black hole entropy: the entropy of a black hole is proportional to the area and not the volume of the event horizon.

Another very useful way of thinking about the holography principle is that it simply says that for a given 3 -volume $V$ in space the state of maximal entropy in nature is given by the largest $B H$ that fits inside $V$, (silently we are making use of the Hawking formula which says that the entropy of a $\mathrm{BH}$ is proportional to the area of its event horizon).

This principle has a deep consequence on perturbative quantum field theory: BH's provide a natural cut-off limit since the above statement says that for fermions for example one cannot have a huge amount of energy concentrated in a tiny region of space because that would collapse into a $\mathrm{BH}$.

There is also a superstring theoretic version of the holography principle using string theory language, the so-called "Maldacena conjecture" which states that string theory on the smooth manifold $A d S_{5} \times S^{5}$ is dual to $N=4 \mathrm{SYM} S U(N)$ gauge theory on the boundary of $A d S_{5}$. In this talk we shall primarily build our understanding of Kontsevich's statement based on this string theoretic version of holography. However let us for the moment go back to the original 't Hooft version of holography and ask:

(Key Question:) What is a $D=d$ CFT? 
In order to answer the above question and eventually understand Kontsevich's statement, we should start by trying to understand the $D=2$ case first. We know from G. Segal what a $D=2$ CFT is, so it seems that somehow we have to generalise his work.

A good motivation to study $D=2$ CFT comes from string theory itself, one can say in fact that $D=2$ CFT is intimately related to string theory: strings are 1-dim objects which in time sweep out 2-manifolds called worldsheets; this is the higher dimensional analogue of the paths (1-dim geometric objects) swept out in time by point particles. However now we are talking about M-Theory in physics which generalises string theory and M-Theory contains the M2 and M5 branes; these are 2 and 5-dim objects respectively whose worldsheets are 3 and 6-dim manifolds. So apart from holography, there is additional motivation coming from M-Theory to understand higher dimensional CFT's: a $D=d$ CFT is the theory describing $(d-1)$-branes.

Now we would like to describe briefly what string theory is classically: basically it is a $\sigma$-model, namely it describes harmonic maps $\phi: \Sigma_{g} \rightarrow X^{10}$ where $\Sigma_{g}$ is a Riemann surface of genus $g$ representing the worldsheets of strings and $X$ is a 26-dim Riemannian manifold with a $B$-field. The dimensionality of $X$ is fixed from consistency arguments (compatibility with special relativity and cancellation of the conformal anomaly). The $B$-field is a real valued 2 -form which is used as a potential to gauge the worldsheets of the strings in order to get our Dirac phase factors; in fact one can think of it as the Poincare Dual (a 2-form) of the worldsheet which is a 2-manifold (it is the analogue of the gauge potentials in Yang-Mills theory, connection 1-forms, although now it has to be a 2-form instead of a 1-form since we are talking about strings whose worlsheets are 2-manifolds whereas for point particles we needed 1-forms because their "worlsheets" were 1-dim objects). This picture needs to include fermions as well in order to be complete but we shall not elaborate more on this. The introduction of fermions along with supersymmetry reduces the dimensionality of $X$ from 26 down to 10 .

The quantum theory of strings is essentially a $D=2 C F T$ (plus a little bit more structure as we shall see later). In order to describe $D=2 \mathrm{CFT}$ one may use the original geometric approach due to G. Segal. This approach however does not lead to the statement of Kontsevich in a straightforward way. Moreover it is not easy to see how it can be generalised to higher 
dimensions which is what we are after. Instead we shall adopt an algebraic approach using the language of operads; in fact we shall see the little 2-discs operad $C_{2}(n)$ arising naturally in our discussion. This is the crucial step in order to understand Kontsevich's statement which is the higher dimensional version of this beautiful fact. The appearence of the operad $C_{2}(n)$ in string theory is not at all obvious and at least for us quite surprising. The link between string theory and the little 2-discs operad $C_{2}(n)$ comes from a deep theorem due to Fred Cohen as we shall try to exhibit shortly. For simplicity we shall restrict our discussion to closed strings. But we shall do that in the last section because we need some mathematical definitions first. The final remark here is that we would still like to generalise Segal's work and get a geometric definition of $D=d$ CFT. Currently this seems out of reach since there are two reasons which make Segal's approach particularly nice for the $D=2$ case (but at the same time act as barriers when trying to generalise into higher dimensions): the conformal group in this case is infinite dimensional and hence contains a lot more information whereas in higher dimensions the conformal group is only finite dimensional. The second reason is that the classification of (compact say) 2-manifolds is simple: compact 2-manifolds are classified by their genus (ie the number of holes) whereas for $D=3$ it is not known if 3 -manifolds can be classified and in dimensions $D \geq 4$ we can only classify simply connected manifolds (in perturbative quantum field theory that means we can only talk about tree level).

\section{A Mathematical Interlude}

In this section now we shall give formal definitions.

\section{Definition 1:}

A Gerstenhaber algebra (or a $G$-algebra) is a graded vector space $V=\oplus_{i \in \mathbf{Z}} V_{i}$ with a dot product $x \cdot y$ defining the structure of a graded commutative associative algebra along with a bracket operation $[x, y]$ of degree -1 defining the structure of a graded Lie algebra such that the bracket is a derivation with respect to the dot product, i.e. it satisfies the Leibniz rule

$$
[x, y \cdot z]=[x, y] \cdot z+(-1)^{(\operatorname{deg}(x)-1) \operatorname{deg}(y)} y \cdot[x, z]
$$

\section{Examples:}

i. Let $A$ be an associative algebra and let $C^{*}(A, A)$ be its Hochschild com- 
plex where $C^{i}(A, A):=\operatorname{Hom}\left(A^{\otimes i}, A\right)$ and the differential $d$ is defined as $\left(x \in C^{n}\right)$ :

$$
\begin{aligned}
(d x)\left(a_{1} \otimes \ldots \otimes a_{n+1}\right):= & a_{1} x\left(a_{2} \otimes \ldots \otimes a_{n+1}\right)+\sum_{i=1}^{n} x\left(a_{1} \otimes \ldots \otimes a_{i} a_{i+1} \otimes \ldots \otimes a_{n+1}\right)+\ldots \\
& +(-1)^{n+1} x\left(a_{1} \otimes \ldots \otimes a_{n}\right) a_{n+1}
\end{aligned}
$$

On the Hochschild complex we can define the usual cup product

$$
\cup: C^{k} \otimes C^{l} \rightarrow C^{k+l}
$$

as follows $\left(x \in C^{k}, y \in C^{l}\right.$ and $\left.a_{i} \in A\right)$ :

$$
(x \cup y)\left(a_{1} \otimes \ldots \otimes a_{k+l}\right):=(-1)^{k l} x\left(a_{1} \otimes \ldots \otimes a_{k}\right) y\left(a_{k+1} \otimes \ldots \otimes a_{k+l}\right)
$$

Moreover we can also define the Gerstenhaber bracket [, ]: $C^{k} \otimes C^{l} \rightarrow C^{k+l-1}$ as:

$$
[x, y]:=x \circ y-(-1)^{(k-1)(l-1)} y \circ x
$$

where

$(x \circ y)\left(a_{1} \otimes \ldots \otimes a_{k+l-1}\right):=\sum_{i=1}^{k-1}(-1)^{i(l-1)} x\left(a_{1} \otimes \ldots \otimes a_{i} \otimes y\left(a_{i+1} \otimes \ldots \otimes a_{i+l}\right) \otimes \ldots \otimes a_{k+l-1}\right)$

The G-bracket gives after a shift of the $\mathbf{Z}$-grading by -1 the structure of a (differentiable graded lie algebra) DGLA on the Hochschild complex. The cup product is not graded commutative (it is only associative) but the induced operation on cohomology is graded commutative. Moreover the Gbracket induces an operation on cohomology which satisfies the Leibniz rule with respect to the cup product, hence the Hochschild cohomology of any associative algebra is in fact a $G$-algebra.

We shall briefly mention three more examples of $G$-algebras:

ii. Polyvector fields on smooth manifolds with wedge product and SchoutenNijenhuis bracket.

iii. Exterior algebra of a Lie algebra with wedge product and extension of the Lie bracket. 
iv. (Rational) homology of double loop space with Pontrjagin product and Samelson bracket.

\section{Definition 2:}

An operad $P$ (of vector spaces) consists of the following data:

a. a collection of vector spaces $P(n), n \geq 0$,

b. an action of the symmetric group $S_{n}$ on $P(n)$ for every $n$,

c. an identity element $i d_{P} \in P(1)$,

d. compositions $m_{\left(n_{1}, \ldots, n_{k}\right)}$

$$
P(k) \otimes\left(P\left(n_{1}\right) \otimes \ldots \otimes P\left(n_{k}\right)\right) \rightarrow P\left(n_{1}+\ldots+n_{k}\right)
$$

for every $k \geq 0$ and $n_{1}, \ldots, n_{k} \geq 0$. These compositions have to be associative, equivariant with respect to the symmetric group actions and the identity element $i d_{P}$ has to satisfy the following naturality property with respect to the composition:

$$
m_{(n)}\left(i d_{P}, p\right)=p
$$

and

$$
m_{(n, 1, \ldots, 1)}\left(p, i d_{P}, \ldots, i d_{P}\right)=p
$$

for all $p \in P(n)$ (one can have a look at [4] for more details).

Example: The "endomorphism operad" of a vector space $V$ is given by $P(n):=\operatorname{Hom}\left(V^{\otimes n}, V\right)$ where the action of the symmetric group and the identity element are the obvious ones and the compositions are defined by the substitutions

$$
\begin{gathered}
\left(m_{\left(n_{1}, \ldots, n_{k}\right)}\left(\phi \otimes\left(\psi_{1} \otimes \ldots \otimes \psi_{k}\right)\right)\right)\left(v_{1} \otimes \ldots \otimes v_{n_{1}+\ldots+n_{k}}\right) \\
:=\phi\left(\psi _ { 1 } ( v _ { 1 } \otimes \ldots \otimes v _ { n _ { 1 } } ) \otimes \ldots \otimes \psi _ { k } \left(v_{n_{1}+\ldots+n_{k-1}+1} \otimes \ldots\right.\right. \\
\left.\left.\otimes v_{n_{1}+\ldots+n_{k}}\right)\right)
\end{gathered}
$$

where $\phi \in P(k):=\operatorname{Hom}\left(V^{\otimes k}, V\right), \psi_{i} \in P\left(n_{i}\right):=\operatorname{Hom}\left(V^{\otimes n_{i}}, V\right)$ and $i=1,2, \ldots, k$.

\section{Definition 3:}

An algebra over an operad $P$ (of vector spaces), or a $P$-algebra, (or equivalently a representation of the operad $P$ ), consists of a vector space $A$ and a collection of multilinear maps $f_{n}: P(n) \otimes A^{\otimes n} \rightarrow A$ for all $n \geq 0$ satisfying the following axioms: 
a. for any $n \geq 0$ the map $f_{n}$ is $S_{n}$-equivariant,

b. for any $a \in A$ we have $f_{1}\left(i d_{P} \otimes a\right)=a$,

c. all compositions in $P$ map to compositions of multilinear operations in $A$.

In other words the structure of an algebra over $P$ on a vector space $A$ is given by a homomorphism from $P$ to the endomorphism operad of $A$.

One can also define modules over algebras over operads.

One can construct operads denoted $\operatorname{Assoc}(n), \operatorname{Lie}(n), \operatorname{Poisson}(n), G(n)$, $A_{\infty}(n), L_{\infty}(n), G_{\infty}(n)$ such that algebras over these operads are associative algebras, Lie algebras, Poisson algebras, Gerstenhaber algebras and their homotopic versions respectively but there is no operad for Hopf algebras (for more details see [4]).

In the definition of operads we can replace our vector space $V$ by a compact topological space $X$ and hence define operads over topological spaces replacing tensor product with Cartesian product.

The analogue of the endomorphism operad will in this case be $P(n):=$ $\left\{\right.$ ContinuousMaps : $\left.X^{n} \rightarrow X\right\}$. Then one can define algebras over topological operads accordingly.

More generally one can define operads and algebras over operads over objects of any symmetric monoidal category $\mathcal{C}$, namely a category endowed with the functor $\otimes: \mathcal{C} \times \mathcal{C} \rightarrow \mathcal{C}$, the identity element $1_{\mathcal{C}} \in \operatorname{Obj}(\mathcal{C})$ and the appropriate coherence isomorphisms for associativity and commutativity of $\otimes$-product.

Operads themselves can be seen as algebras over the coloured operad. (see [8] p.12 Remark 1.)

In particular we would like to consider operads in the symmetric monoidal category Complexes whose objects are $\mathbf{Z}$-graded complexes of abelian groups and arrows morphisms of complexes. These are called differential graded operads or $d g$-operads for short. So each component $P(n)$ of an operad of complexes will be a complex, namely a vector space decomposed into a direct sum $P(n)=\oplus_{i \in \mathbf{Z}} P(n)^{i}$ and endowed with a differential $d: P(n)^{i} \rightarrow P(n)^{i+1}$ of degree +1 such that $d^{2}=0$. Then every dg-operad $P$ has a corresponding homology operad denoted $H_{*}(P)$. 


\section{Key idea:}

There is a natural way to construct an operad of complexes from a topological operad by using essentially the singular chain complex of topological spaces.

Let $d \geq 1$ be an integer. Denote by $G_{d}$ the $(d+1)$-dimensional Lie group of affine transformations acting on $\mathbf{R}^{\mathbf{d}}$ via $u \mapsto \lambda u+v$ where $\lambda>0$ is a real number and $v \in \mathbf{R}^{\mathbf{d}}$ is a vector. This group acts simply transitively on the space of closed discs in $\mathbf{R}^{\mathbf{d}}$ and the disc with centre $v$ and radius $\lambda$ is obtained from the standard disc

$$
D_{0}:=\left\{\left(x_{1}, \ldots, x_{d}\right) \in \mathbf{R}^{\mathbf{d}} \mid x_{1}^{2}+\ldots+x_{d}^{2} \leq 1\right\}
$$

by a transformation from $G_{d}$ with parameters $(v, \lambda)$.

\section{Definition 4:}

The little d-discs operad $C_{d}(n)$ is a topological operad with the following structure:

a. $C_{d}(0):=\emptyset$,

b. $C_{d}(1):=*$,

c. for $n \geq 2$ the space $C_{d}(n)$ is the space of configurations of $n$ disjoint $d$-discs $\left(D_{i}\right)_{1 \leq i \leq n}$ inside the standard $d$-disc $D_{0}$.

The composition

$$
C_{d}(k) \times C_{d}\left(n_{1}\right) \times \ldots \times C_{d}\left(n_{k}\right) \rightarrow C_{d}\left(n_{1}+\ldots+n_{k}\right)
$$

is obtained by applying elements from $G_{d}$ associated with discs $\left(D_{i}\right)_{1 \leq i \leq n}$ in the configuration in $C_{d}(k)$ to configurations in all $C_{d}\left(n_{i}\right), i=1,2, \ldots, k$ and putting the resulting configurations together. The action of the symmetric group $S_{n}$ on $C_{d}(n)$ is given by renumerations of indices of discs $\left(D_{i}\right)_{1 \leq i \leq n}$.

Remark: The little d-discs operad $C_{d}(n)$ was introduced by Peter May (see [13]) and Boardmann-Vogt (see [14]), in the late 70's in order to describe homotopy types of $d$-fold loop spaces, namely spaces of continuous maps

$$
\operatorname{Maps}\left(S_{+}^{d}, X_{+}\right)
$$

where "+" denotes base point, $X$ is a topological space, $S^{d}$ is the $d$-dim sphere. The little $d$-discs operad is the most important operad in homotopy 
theory.

The key result relating the little $d$-discs operad $C_{d}(n)$ with $d$-fold loop spaces is that (with field coefficients) chains of $d$-fold loop spaces become naturally $d$-algebras i.e. algebras over the operad Chains $_{d}(n)$. (In fact the above statement is true even without taking "chains" in both sides).

We have the following well-known

Fact: The space $C_{d}(n)$ is homotopy equivalent to the configuration space of $n$ pairwise distinct points in $\mathbf{R}^{\mathbf{d}}$ :

$$
\mathbf{F}\left(n, \mathbf{R}^{\mathbf{d}}\right):=\left(\mathbf{R}^{\mathbf{d}}\right)^{n}-\operatorname{Diag}=\left\{\left(v_{1}, \ldots, v_{n}\right) \in\left(\mathbf{R}^{\mathbf{d}}\right)^{n}\right.
$$

$\mid v_{i} \neq v_{j}$ for $\left.i \neq j\right\}$

\section{Definition 5:}

Let $\tilde{C_{d}}(n):=\mathbf{F}\left(n, \mathbf{R}^{\mathbf{d}}\right) / G_{d}$ which is also the Fulton-MacPherson operad $F M_{d}(n)$.

For $n=2, F M_{d}(2)$ is homotopy equivalent to the $(d-1)$-sphere $S^{d-1}$. For all $n \geq 3, F M_{d}(n)$ is a manifold with corners which can be identified explicitly.

\section{Definition 6:}

For $d \geq 0$, a $d$-algebra is an algebra over the operad Chains $\left(C_{d}\right)$ in the category of complexes.

One then has:

Theorem 1. (F. Cohen, see [6]).

There is a natural homotopy equivalence

$$
G(n) \simeq H_{*}\left[\operatorname{Chains}_{2}(n)\right]
$$

Recall the fact that the Hochschild cohomology of any associative algebra has a natural $G$-algebra structure. The original Deligne conjecture (see [3]), was that the Hochschild complex of an associative algebra (or more generally the Hochschild complex of an $A_{\infty}$-algebra as mentioned in [8]) itself carries a natural 2-algebra structure, i.e. it has an action of the 
operad Chains $_{2}(n)$. Its higher dimensional version due to Kontsevich says:

"For any $d$-algebra there is a natural action of a universal (in an appropriate sense defined up to homotopy) $(d+1)$ - algebra".

Useful facts:

$$
\begin{aligned}
& \operatorname{Assoc}(n) \simeq H_{*}\left[\text { Chains }_{1}(n)\right] \\
& \operatorname{Lie}(n) \simeq H_{n-1}\left[\text { Chains }_{2}(n)\right]
\end{aligned}
$$

Since in general homotopic versions of various algebras appear when the product is originally defined on the cohomology and one wants to "lift" the structure to the cochain level, one has the following general relations between algebras and their "homotopic versions":

$$
\begin{gathered}
\operatorname{Assoc}(n) \simeq H_{*}\left[A_{\infty}(n)\right] \\
\operatorname{Lie}(n) \simeq H_{*}\left[L_{\infty}(n)\right] \\
G(n) \simeq H_{*}\left[G_{\infty}(n)\right]
\end{gathered}
$$

Aside: The above discussion was about the little discs operad and based loop spaces. One also has a variation of the above, the so called framed little $d$-discs operad denoted $C_{d}^{f}(n)$ which is related to free loop spaces. The framed little 2-discs operad $C_{2}^{f}(n)$ is homotopic to the cactus operad and the (rational) homology of the framed little 2-discs operad is homotopic to the BV-operad. The main result is then that the (rational) homology of the free loop space $H_{*}(L X)$ where $X$ is a compact oriented manifold (after an apropriate shift) has a BV-algebra structure, namely it is an algebra over the Batalin-Vilkovisky operad. At this point we would like to remind the reader of the fact that $B V$-algebras appear in the Lagrangian formalism of field theories whereas Gerstenhaber algebras appear in BRST cohomology which is Hamiltoniam formalism of a field theory. For more details on the cactus operad, free loops and BV algebras we refer to [10].

Then the second important result is the following

Theorem 2: (D. Tamarkin 1998, see [9]).

In characteristic zero, the operad $\operatorname{Chains}\left(C_{d}\right) \otimes \mathbf{R}$ is formal, i.e. it is homotopy equivalent to its corresponding homology operad. 


\section{The appearence of the operad $C_{2}(n)$ in string theory}

After giving all these mathematical definitions we now return back to physics. G. Segal (see [15]), defined a $D=2 \mathrm{CFT}$ as roughly a topological vector space $H_{S^{1}}$ and to each cobordism (which physically represents a Feynman diagram for strings which are 1-dim objects with 2-dim worldsheets) $\Sigma_{g}: S^{1} \rightsquigarrow S^{1}$ we associate an operator $U_{\Sigma_{g}}$ where $\Sigma_{g}$ is a Riemann surface of genus $g$ with a conformal class on it. We can replace the boundary of $\Sigma_{g}$ with more copies of $S^{1}$ representing more than one incoming and outgoing closed strings. The case $g=0$ corresponds to tree-level in physics.

The space of maps $U_{\Sigma_{g}}$ is parametrised by $A:=\operatorname{Conf}\left(\Sigma_{g}\right) / \operatorname{Diff}\left(\Sigma_{g}, \partial \Sigma_{g}\right)$ and $A$ acts on $H_{S^{1}}$ where the denominator denotes diffeomorphisms which become the identity on the boundary. $A$ is only a semigroup under concatenation but it has a Lie algebra $\operatorname{Vect}\left(S^{1}\right)_{\mathbf{C}}$ and its fundamental group is Z. We have a composition law on $H_{S^{1}}$ for every conformal structure on $\Sigma_{g}$ which is associative up to the action of $A$.

More concretely we use Lagrangian formalism and write

$$
U_{\Sigma_{g}}=\int_{\phi: \Sigma_{g} \rightarrow \mathbf{R}} e^{-S(\phi)} \mathcal{D} \phi
$$

where

$$
S(\phi):=\frac{1}{2} \int_{\Sigma_{g}} d \phi \wedge * d \phi
$$

If we change the conformal class on $\Sigma_{g}$ by $f$, then $U_{\Sigma_{g}}$ is multiplied by the factor $e^{c L(f)}$ where $c$ is the central charge and $L(f)$ is given by the Liouville formula

$$
L(f)=\int_{\Sigma_{g}}\left[\frac{1}{2} d f \wedge * d f+f K G+\frac{1}{2}\left(e^{2 f}-1\right) \omega_{G}\right]
$$

where $G$ is the metric with Gauss curvature $K$ and volume form $\omega_{G}$. Moreover $H_{S^{1}}$ carries a projective representation of the cobordism category, thus each cobordism $\Sigma_{g}$ has associated to it a complex line bundle (in its most general form these are tensor products of determinant line bundles 
which generalise the usual basis $L_{n}=e^{i h \theta} \frac{d}{d \theta}$ of $\left.\operatorname{Vect}\left(S^{1}\right)_{\mathbf{C}}\right)$.

This geometric picture of G. Segal is very nice but when trying to generalise it in higher dimensions one faces problems.

Now we shall modify Segal's definition using the more convenient language of operads, we follow [4]. We shall swich to Hamiltonian formalism and point out that our formalism below is less satisfactory than G. Segal's because it works only for genus 0 Riemannian surfaces but it is easier to generalise in higher dimensions. We shal explain in detail the structures appearing in the $D=2$ case and then we shall try to see how much can be immediately generalised to higher dimensions.

Before doing that we would like to make a comment: there are two ways to construct operads related to Riemann surfaces: the first one is by using moduli spaces of punctured Riemann surfaces and compactify them, such operads are roughly denoted $M(n)$; the second is by decorating the puctures with local coordinates. One can sew two Riemann surfaces together unambiguously (up to modular equivalence) by using suitable local coordinates.

Let $R(n)$ be the moduli space of nondegenerate Riemann spheres $\Sigma$ with $n$ labelled punctures and non-overlapping holomorphic discs at each puncture (holomorphic embeddings of the standard disc $|z|<1$ to $\Sigma$ centered at the puncture). The spaces $R(n), n \in \mathbf{N}^{*}$ form an operad under sewing Riemann spheres at punctures (cutting out the discs $|z| \leq r$ and $|w| \leq r$ for some $r=1-\epsilon$ at sewn punctures and identifying the annuli $r<|z|<1 / r$ and $r<|w|<1 / r$ via $w=1 / z)$. The symmetric group interchanges punctures along with the holomorphic discs.

Consider the complexification $V$ of the Virasoro algebra of complex valued vector fields on the circle, generated by elements $L_{m}=z^{m+1} \partial / \partial z$, $m \in \mathbf{Z}$, with the commutators $\left[L_{m}, L_{n}\right]=(n-m) L_{m+n}$. Then one has:

\section{Definition 7:}

A $D=2$ conformal field theory at tree level consists of the following data: 1. A topological vector space $H$ called state space.

2. An action $T: V \otimes H \rightarrow H$ of the Virasoro algebra $V$ on $H$.

3. A vector $\mid \Sigma>\in H o m\left(H^{\otimes n}, H\right)$ for each $\Sigma \in R(n)$ depending smoothly on $\Sigma$. These data must satisfy the following relations: 
4. $T(\underline{v})|\Sigma>=| \delta(\underline{v}) \Sigma>$, where $\underline{v}=\left(v_{1}, \ldots, v_{n}\right) \in V$ and $\delta$ is the natural action of $V^{n}$ on $R(n)$ by infinitesimal reparametrisations at punctures. In particular $T(\underline{v}) \mid \Sigma>=0$ whenever $\underline{v}$ can be extended to a holomorphic vector field on $\Sigma$ outside of the discs.

5. The correspondence $\Sigma \mapsto|\Sigma\rangle$ defines the structure of an algebra over the operad $R(n)$ on the space of states $H$.

So briefly, the slogan is that a $D=2$ CFT is an algebra over the operad $R(n)$.

\section{Definition 8:}

A string background (at the tree level) is a $D=2 \mathrm{CFT}$ based on the vector space $H$ with the following additional data:

1. A Z Zrading $H=\oplus_{i \in \mathbf{Z}} H_{i}$ on the state space.

2. An action of the Clifford algebra $C\left(V \oplus V^{*}\right)$ which is denoted $b: V \otimes H \rightarrow$ $H$ and $c: V^{*} \otimes H \rightarrow H$ for generators of the Clifford algebra, the degree of $b$ is -1 and the degree of $c$ is +1 .

3. A differential $Q: H \rightarrow H, Q^{2}=0$, of degree +1 called BRST operator satisfying $Q b+b Q=T$.

The differential graded complex $(H, Q)$ is called the BRST complex and the degree is called the ghost number.

One of the nicest implications of a string background is the construction of a morphism of complexes $\omega_{n}: \operatorname{Hom}\left(H, H^{\otimes n}\right) \rightarrow \Omega^{*}(R(n))$, from the complex of linear mappings between tensor powers of the BRST complex $H$ to the de Rham complex of the space $R(n)$.

Taking the cohomology of the BRST complex gives the space of physical states. In physics this amounts to mod out gauge invariance (this is a cohomological approach to symplectic reduction in the case of a symplectic manifold carrying a Lie group action which is more convenient in infinite dimensions, i.e. field theory).

Then a closed string field theory is a string background together with a morphism of operads $\psi: M(n) \rightarrow R(n)$. This however does not readily generalise to higher dimensions since both operads $M(n)$ and $R(n)$ are related to moduli spaces of Riemann surfaces. The aspect of closed string field theory which will be useful for higher dimensional generalisations is the 
following:

Key fact in physics: The space of physical states in closed string field theory (namely BRST cohomology) has the structure of a Gerstenhaber algebra.

Let us explain this a little bit more: from the work of G. Segal we knew that in general $D=2$ topological quantum field theories (and in particular closed string field theory) are $\mathbf{Z}$-graded commutative associative Frobenious algebras, hence they are graded associative algebras. Yet it was observed by Witten and Zwiebach that closed string field theory also carries the structure of a Differential Graded Lie Algebra relative to another grading which is "the associative grading - 1". In fact these 2 structures can be combined together to give a $G$-algebra structure.

Now we make use of Cohen's theorem saying that $G$-algebras correspond exactly to the homology of the chains of the little 2-discs operad and of Tamarkin's result to deduce that the space of physical states of closed string field theory (which is a special case of $D=2$ CFT) has a natural (defined up to homotopy) 2-algebra structure.

So this is the important relation between strings and the operad $C_{2}(n)$ that plays the fundamental role to understand Kontsevich's statement which is then simply the higher dimensional version of the above fact which originally holds for strings ( $D=2$ case).

\section{Discussion}

Let us start with some

\section{Remarks:}

1. The original Deligne conjecture, namely the case $d=1$, was proved by Kontsevich and Soibelman in 2000 using ideas and techniques from Dan Quillen's homotopical algebra (see [5]), which roughly is a non-linear generalisation of homological algebra. 
2. As Kontsevich explains in his article, from Deligne's conjecture and from Tamarkin's theorem (namely the formality of the operad $C h a i n s C_{d}(n)$ ), follows almost immediately his earlier result on deformation quantization of symplectic (Poisson) manifolds for the case where the associative algebra of interest is just the polynomial algebra in $n$ variables

$$
A:=\mathbf{R}\left[x_{1}, \ldots, x_{n}\right] .
$$

Let us recall that Kontsevich's result was that for the associative algebra $A$ above one has that its Hochschild complex $C^{*}(A, A)$ is homotopic as a Lie algebra to its Hochschild cohomology $H^{*}(A, A)$. An equivalent statement is that for the Euclidean space $X=\mathbf{R}^{n}$, the Hochschild complex of the associative algebra of functions on $X$ equipped with the Gerstenhaber bracket is homotopic as DGLA to the $\mathbf{Z}$-graded superalgebra of polyvector fields on $X$ equipped with the Schouten-Nijenhuis bracket.

3. The structure of an $A_{\infty}$-algebra has appeared recently in open strings. Moreover let us mention that one of the main examples of homotopy associative algebras (or $A_{\infty}$-algebras) is singular chains of based loops.

Perhaps here we should mention another important result: the BRST complex of closed string field theory has also the structure of a $G_{\infty}$-algebra (see [4]). That makes someone to speculate on a relation between the operads $G_{\infty}(n)$ and Chains $_{2}(n)$. For example it is unknown if double loop spaces (which are the primary examples of 2-algebras) also carry a homotopy Gerstenhaber algebra structure.

Now let us try to answer the following question: Why should physics care about the Deligne conjecture? We think for 2 reasons:

i. The fact that the Hochschild complex of an associative algebra is a 2-algebra (original conjecture) is related to the action of the GrothendieckTeichmuller $(G-T)$ group. The fact that the Hochschild complex plays the fundamental role in the theory of deformations of associative algebras explains why its study is important in quantum field theory if one adopts the deformation quantization approach.

In other words the goal is to understand the action of the G-T group on the space of all deformation quantizations on the associative algebra of 
functions (in fact one needs a little more structure, i.e. a Poisson algebra structure) on a given (spacetime or phase space respectively) manifold and this is believed to be related to gauge symmetry. Yet all these are far from being clear at the moment. Let us briefly recall that the GrothendieckTeichmuller group can be defined as the automorphism group of the tower of the pro-nilpotent completions of the pure braid groups; the pure braid group of $n$ strings is the fundamental group of the configuration space of $n$ points in the plane $\mathbf{F}\left(n, \mathbf{R}^{\mathbf{2}}\right)$.

ii. Let us forget deformation quantization now and let's focus on holography: If one wants to understand $(d+1)$-dim quantum gravity, one approach is to follow holography. This means that equivalently one should understand $D=d$ CFT. From the string theory example we have learnt that $D=2$ CFT is related to 2-algebras. It is then reasonable to expect that $D=d$ CFT should be related to $d$-algebras. So the hope is that this higher dimensional Deligne conjecture will tell us something about the "BRST complex" whose cohomology would describe the $D=d$ CFT. So the $D=d$ CFT is a $d$-algebra and following the Hamiltonian formalism the space of physical states will be expresseed as a cohomology; then the Deligne conjecture will contain information about the cochain level of the cohomology. This information at the cochain level has a nice geometric interpretation as follows (if one adopts the Lagrangian formalism of the theory):

Holography tells us that $(d+1)$-quantum gravity is reduced to some (conformal) field theory on the boundary. Obviously there are many $(d+1)$ quantum gravity theories which will have the same boundary theory, simply because there are many $(d+1)$-manifolds with the same $d$-manifold as boundary. Consider all $(d+1)$-bulk theories then with the same boundary $d$-theory as some sort of an extra "gauge freedom", ie that they define a "fibre" over the fixed boundary $d$-theory. Equivalently, we have a whole cobordismm class of a boundary theory consisting of bulk theories which, because of holography, are physically the same theory. Assuming that the boundary theory is described by a $d$-algebra, we know that the Grothendieck-Teichmuller group acts in an analogous way that the gauge group acts in ordinary gauge theories (the fibres are essentially the gauge orbits). The higher dimensional Deligne conjecture is another way to express this action of the Grothendieck-Teichmuller group which takes care of this extra gauge freedom arising from the fact that many $(d+1)$-bulk theories have the same resulting boundary $d$-theory. This is roughly the statement 
that the Hochschild complex of a $d$-algerba has a natural (appropriately defined up to homotopy) $(d+1)$-algebra structure.

Yet this is not still the end of the story: the action of the G-T group is closely related to the action of the motivic group: since the little $d$-discs operad is formal, we can form a torsor; in general given any pair of equivalent objects $A$ and $B$ in a category, we can form the space of all isomorphisms between the objects $A$ and $B$ denoted $I s o(A, B)$. The groups of automorphisms of both $A$ and $B$ denoted $A u t(A)$ and $A u t(B)$ respectively act naturally on $I s o(A, B)$ and their actions commute. A torsor is a structure that encodes this information. If we think of the formality of the operad $C_{d}(n)$ as defining a pair of equivalent objects in the category of $\mathbf{Z}$-graded complexes, we can indeed define a torsor and then the action of the G-T group will play the role of say $A u t(A)$ whereas the motivic group wil play the role of $A u t(B)$. Further evidence that our proposal to use $d$-algebras to describe $D=d$ CFT is not at all unreasonable comes from the fact that Tamarkin in order to prove his formality theorem he made use of the Drinfeld-Kazhdan associator whose origin is the Knizhnik-Zamolodchikov equations, namely it comes directly from $D=2 \mathrm{CFT}$ ! (For the precise definitions of the motivic group and the Drinfeld-Kazhdan associator we refer to [7]).

Going back to physics a pessimist might argue that the holography principle does not really "resolve" the BH paradoxe; it simply pushes it even further to the realm of quantum gravity which is terra incognita for today's physics. We however would like to adopt a more positive point of view: by accepting the validity of holography we can actually use it in order to learn something about quantum gravity and at the same time we try to understand its wider implications for physics. Finally we would like to mention that there have been already some positive tests for the validity of holography mainly in the framework of some calculations related to its string theoretic version (Maldacena conjecture).

Operads (and the little discs operad in particular) currently should be seen more as an algebraic framework to conceptually understand higher dimensional CFT's. As a computational device in $D=2$ CFT people use Topologiacl Vertex Operator Algebras (TVOA) as a tool to construct topological CFT but again their higher dimensional generalisation is not obvious.

The final comment we would like to make is the following: the structure 
of a Gerstenhaber algebra which was observed in $D=2$ CFT ment that the dot product (which gives the OPE) and the Lie bracket (which gives the conformal symmetry) are combined in a "nice way" in the space of physical states (ie that the bracket is a derivation with respect to the product). Our proposal that higher dimensional CFT's are described by $d$-algebras means that roughly we still have this nice combination in higher dimensions between the dot product and the Lie bracket. However in general a Gerstenhaber algebra "does not care" whether the Lie bracket comes specifically from conformal symmetry. Thus our proposal looks more like an algebraic framework to understand higher dimensional CFT and holography for the moment; however if we want to make its relation with physics - with conformal symmetry in particular - more concrete and if we want to properly and fully justify the proposal that a $d$-algebra should describe $D=d \mathrm{CFT}$, we would have to add more specific information about conformal symmetry (Lie bracket) and dot product (OPE) in higher dimensions. So it seems that the problem is not whether our proposal is valid but rather that it may be far too general to be of any practical use. In any case it helps conceptually we believe. For example one desired property would be to consider representations of the operad $C_{d}(n)$ on the endomorphism operad of a Clifford algebra (and not just a vector space) in order to make the theory chiral. However for the moment we do not know how to incorporate the Cardy condition in our formalism.

\section{Acknowledgements:}

The author would like to thank Maxim Kontsevich and Graeme Segal for useful discussions. This work was partly supported financially by an E.P.S.R.C. research grant (contract No: GR/R64995/01). An earlier version of this article under the title "Holography and the Deligne Conjecture" was presented as a poster/oral presentaton during the XIVth International Congress of Mathematical Physics, Lisbon, Portugal, 28 July-2 Agust 2003.

\section{References}

[1] S.R. Das and S.D. Mathur: "The Quantum Physics of Black Holes: Results from String Theory", Annual Review of Nuclear and Particle 
Science Vol 50 (2000) and gr-qc/0105063.

[2] G. 't Hooft: "Dimensional Reduction in Quantum Gravity", Essay dedicated to Abdus Salam in "Salamfestschift: a collection of talks", editors: A. Ali, J. Ellis and S. Randjbar-Daemi, World Scientific 1993 and gr-qc/9310026.

[3] P. Deligne, Ph. Griffiths, J. Morgan, D. Sullivan: "Real homotopy theory of kahler manifolds", Invent. Math. 29 (1975).

[4] J-L. Loday, J.D. Stasheff and A.A. Voronov: "Operads: Proceedings of Renaissence Conferences", Contemp. Maths. 202 AMS 1997.

[5] D.G. Quillen: "Homotopical Algebra", Lect. Notes Math., Vol 43, Springer 1967.

[6] F.R. Cohen: The homology of $C_{n+1}$-spaces, $n \geq 0$, The homology of iterated loop spaces", Lect. Notes in Math., vol 533, Springer (1976).

[7] M. Kontsevich: "Operads and Motives in Deformation Quantization", Lett. Math. Phys. 48.1 (1999) and QA/9904055.

[8] M. Kontsevich and Y. Soibelman: "Deformations of algebras over operads and Deligne's conjecture", Conference Moshe Flato 1999, Vol I, Math. Phys. Studies 21, Kluwer (2000), 225-308 and QA/0001151.

[9] D. Tamarkin: "Another Proof of M. Kontsevich formality theorem", math-qa/9803025.

D. Tamarkin: "Deformation complex of a d-algebra is a $(d+1)$-algebra", math-qa/0010072. 
D. Tamarkin: "Action of the Grothendieck-Teichmuller Group on the operad of Gerstenhaber Algebras", math-qa/0202039.

[10] A.A. Voronov: "Notes on Universal Algebra", preprint QA/0111009.

M. Gerstenhaber, A. Voronov: Homotopy G-algebras and moduli space operad", Intern. Math. Res. Notices No 3 (1995).

[11] I. P. Zois: "A new invariant for $\sigma$-models", Commun. Math. Phys. 209.3 (2000).

[12] I. P. Zois: "Black Hole Entropy, Topological Entropy and the BaumConnes Conjecture in K-Theory", Jour. Phys. A:Math. 35.12 (2002).

[13] J. P. May: "Infinite Loop Space Theory", Bull. Amer. Math. Soc. 83.4 (1977).

[14] J.M. Boardmann, R. Vogt: "Homotopy invariant algebraic structures on topological spaces", Lect. Notes in Math. 347, Springer (1973).

[15] G. Segal: "Topological and Conformal Field Theories", Graduate Lecture Course, Mathematical Institute, Oxford University, UK, Hilary Term 2000.

[16] P. K. Townsend: "Black Holes", Lecture Notes, DAMTP, Cambridge University, UK, 1998. 